\title{
Transcriptomic analysis of caecal tissue in inbred chicken lines that exhibit heritable differences in resistance to Campylobacter jejuni
}

Kay M. Russell ${ }^{1}$, Jacqueline Smith ${ }^{1}$, Abi Bremner ${ }^{1}$, Cosmin Chintoan-Uta ${ }^{1}$, Lonneke Vervelde ${ }^{1}$, Androniki Psifidi ${ }^{2}$ and Mark P. Stevens ${ }^{1 *}$

\begin{abstract}
Background: Campylobacter jejuni is the leading cause of bacterial gastroenteritis in humans and the handling or consumption of contaminated poultry meat is a key source of infection. Selective breeding of poultry that exhibit elevated resistance to Campylobacter is an attractive control strategy. Here we studied the global transcriptional response of inbred chicken lines that differ in resistance to $C$. jejuni colonisation at a key site of bacterial persistence.

Results: Three-week-old chickens of line $\sigma_{1}$ and $N$ were inoculated orally with C. jejuni strain M1 and caecal contents and tonsils were sampled at 1 and 5 days post-infection. Caecal colonisation was significantly lower in line $\sigma_{1}$ compared to line $N$ at 1 day post-infection, but not 5 days post-infection. RNA-Seq analysis of caecal tonsils of both lines revealed a limited response to C. jejuni infection compared to age-matched uninfected controls. In line $\mathrm{N}$ at days 1 and 5 post-infection, just 8 and 3 differentially expressed genes (DEGs) were detected (fold-change $>2$ and false-discovery rate of $<0.05$ ) relative to uninfected controls, respectively. In the relatively resistant line $\sigma_{1}$, a broader response to C. jejuni was observed, with 69 DEGs relating to immune regulation, cell signalling and metabolism at 1 day post-infection. However, by day 5 post-infection, no DEGs were detected. By far, the greatest number of DEGs were between uninfected birds of the two lines implying that differential resistance to $C$. jejuni is intrinsic. Of these genes, several Major Histocompatibility Complex class I-related genes (MHCIA1, MHCBL2 and MHCl Y) and antimicrobial peptides (MUC2, AvBD10 and GZMA) were expressed to a greater extent in line N. Two genes within quantitative trait loci associated with C. jejuni colonisation were also more highly expressed in line N (ASIC4 and BZFP2). Quantitative reverse-transcriptase PCR analysis of a subset of transcripts confirmed the RNA-Seq results.
\end{abstract}

Conclusions: Our data indicate a limited transcriptional response in the caecal tonsils of inbred chickens to intestinal colonisation by Campylobacter but identify a large number of differentially transcribed genes between lines $\sigma_{1}$ and $N$ that may underlie variation in heritable resistance to $C$. jejuni.

Keywords: Campylobacter jejuni, Chicken, Resistance, Susceptibility, Transcriptome, Gene expression, Inbred, RNASeq

\footnotetext{
* Correspondence: Mark.Stevens@roslin.ed.ac.uk

'The Roslin Institute and Royal (Dick) School of Veterinary Studies, University of Edinburgh, Easter Bush, Midlothian EH25 9RG, UK

Full list of author information is available at the end of the article
}

(c) The Author(s). 2021 Open Access This article is licensed under a Creative Commons Attribution 4.0 International License, which permits use, sharing, adaptation, distribution and reproduction in any medium or format, as long as you give appropriate credit to the original author(s) and the source, provide a link to the Creative Commons licence, and indicate if changes were made. The images or other third party material in this article are included in the article's Creative Commons licence, unless indicated otherwise in a credit line to the material. If material is not included in the article's Creative Commons licence and your intended use is not permitted by statutory regulation or exceeds the permitted use, you will need to obtain permission directly from the copyright holder. To view a copy of this licence, visit http://creativecommons.org/licenses/by/4.0/ The Creative Commons Public Domain Dedication waiver (http://creativecommons.org/publicdomain/zero/1.0/) applies to the data made available in this article, unless otherwise stated in a credit line to the data. 


\section{Background}

Campylobacter is estimated to have caused 95 million cases of acute gastroenteritis in humans in 2010, with the loss of 21,000 lives and 2.1 million disabilityadjusted life years [1]. In the United Kingdom alone, 63, 946 laboratory-confirmed cases of human campylobacteriosis were recorded in 2017 [2] and a further 9.3 cases were predicted to be unreported for every one captured by national surveillance [3]. Such infections have been estimated to cost the UK economy approximately $£ 50$ million per annum through lost productivity and healthcare costs [4]. Campylobacter infections in humans often involve watery diarrhoea, abdominal cramps and nausea but generally resolve within a week [5]. However, infections can relapse and severe sequelae exist, including inflammatory neuropathies such as the Guillain-Barré syndrome [5].

Source attribution studies unequivocally implicate the handling or consumption of contaminated poultry meat as a key risk factor for human campylobacteriosis, with up to $80 \%$ of cases thought to be attributable to the avian reservoir [6, 7]. The caeca are a key site of persistence of Campylobacter in poultry, where numbers of $C$. jejuni can reach as high as $10^{10}$ colony forming units (CFU)/g of contents in the absence of overt pathology. Given such levels, contamination of carcasses with numbers of $C$. jejuni predicted to be adequate for human infection is challenging to prevent during the slaughter process [8]. A recent survey in the United Kingdom found that $54 \%$ of fresh retail chicken was contaminated with Campylobacter [9]. Birds generally become colonized with $C$. jejuni from their environment, and across Europe the prevalence of $C$. jejuni positive flocks ranges from 18 to $90 \%$, with seasonal variation and the highest levels occurring in the summer months [10]. Control of Campylobacter in poultry primarily relies on stringent on-farm biosecurity measures and carcass treatments. No commercial vaccines exist and it is likely that a multifactorial approach will be required.

It has been estimated that a $2 \log 10$ reduction in the level of poultry carcass contamination by $C$. jejuni could lower the incidence of human cases due to this source by 12 to 30-fold [11]. However, more modest estimates suggest a $3 \log _{10}$ reduction in caecal colonisation would reduce human cases by $58 \%$, although with a high degree of uncertainty [12]. One option to achieve this is to improve the intrinsic resistance of chickens to Campylobacter colonisation. Differences exist in the levels of colonisation across and within commercial broiler lines $[13,14]$ and these have been associated with variation in the transcriptome of the caeca [14-16] and spleen [17]. Genome-wide association studies in a commercial broiler population have indicated that resistance to caecal C. jejuni colonisation is under moderate genetic control [14]. However, heritable differences in resistance have been associated with quantitative trait loci (QTL) $[14,18]$, and the transcription of genes related to immunity $[14,19]$.

White Leghorn-derived inbred chicken lines $6_{1}$ and $N$ have been reported to be relatively resistant and susceptible to colonisation by several $C$. jejuni strains, respectively [20, 21], with F1 progeny of a cross exhibiting intermediate phenotypes [20]. Genome-wide association studies using backcross $\left[\left(6_{1} \times \mathrm{N}\right) \times \mathrm{N}\right]$ and ninth generation advanced intercross $\left(6_{1} \times \mathrm{N}\right)$ populations have identified QTLs associated with resistance to caecal colonisation by C. jejuni in these lines [21]. In this study, two candidate genes were identified in the QTL regions, ASIC4, located on chromosome 7, and BZFP2 located on chromosome 16, indicating a potential association with the Major Histocompatibility Complex (MHC) locus also present on chromosome 16. Immune-related genes such as IL6, CXCLi2 and CCLi2 [22] and immune-related pathways including lymphocyte activation, cytokine signalling and Ig production $[15,17,19]$ have also been proposed to contribute to the differential resistance of chicken lines. Irrespective of the association of genes or expression patterns with heritable resistance, a need exists to better understand how birds respond to C. jejuni during infection, where previous studies have suggested a pro-inflammatory response that is limited in magnitude and timing [23-26], but which may also differ between commercial broiler lines [27].

Line $6_{1}$ and $\mathrm{N}$ chickens not only differ in resistance to Campylobacter, but to gut colonisation by Salmonella enterica serovar Typhimurium and genetic associations have been mapped using a backcross [28]. We recently demonstrated that limited differences exist between lines $6_{1}$ and $\mathrm{N}$ in their caecal microbiota and reciprocal transplants of caecal microbiota did not alter their resistance to $C$. jejuni colonisation, suggesting a role for host factors [29]. Here, we used RNA-Seq to investigate the caecal transcriptome of line $6_{1}$ and $N$ chickens, both in uninfected birds to identify differences between the lines that may underlie differential resistance to pathogens and following experimental challenge with C. jejuni.

\section{Results}

Challenge of line $6_{1}$ and line $\mathrm{N}$ birds with C. jejuni M1 confirms differential resistance early after inoculation

To examine the level of resistance and susceptibility of lines $6_{1}$ and $\mathrm{N}$ to colonisation by $C$. jejuni M1, threeweek-old birds from each line were challenged with $10^{8}$ CFU of $C$. jejuni M1 and the resulting numbers of $C$. jejuni in the caecal content determined at 1 and 5 days post-infection (dpi). At $1 \mathrm{dpi}$, line $6_{1}$ birds exhibited a significantly $(P<0.01)$ lower level of $C$. jejuni colonisation in the caeca compared to line $\mathrm{N}$ by approximately 3 
$\log _{10} \mathrm{CFU} / \mathrm{g}$ (Fig. 1). At $5 \mathrm{dpi}$, no significant difference in caecal colonisation by $C$. jejuni was observed between the two lines. These results indicate that line $6_{1}$ is relatively resistant to $C$. jejuni $\mathrm{M} 1$ during early colonisation, as reported for other strains [20,21,29].

\section{Transcriptional responses to infection in chicken lines differing in $C$. jejuni resistance}

To explore transcriptomic differences underlying the relative resistance of line $6_{1}$ and susceptibility of line $\mathrm{N}$ to $C$. jejuni M1 colonisation, RNA-Seq analysis was performed on caecal tonsil tissue from both infected and age-matched uninfected control birds of both lines. Birds were inoculated with $C$. jejuni at 3 weeks-of-age for parity with earlier reports on differential resistance to $C$. jejuni at this age [20,21]. Differentially expressed genes (DEGs) were identified between experimental groups as follows: (1) line $\mathrm{N}$ infected vs control birds at $1 \mathrm{dpi}$, (2) line $\mathrm{N}$ infected vs control birds at $5 \mathrm{dpi}$, (3) line $6_{1}$ infected vs control birds at $1 \mathrm{dpi}$, (4) line $6_{1}$ infected vs control birds at $5 \mathrm{dpi}$ and (5) line $\mathrm{N}$ vs line $6_{1}$ control birds from both 1 and 5 dpi due to the high similarity between control samples across both time points, identified by sample clustering analysis. Gene Ontology (GO) analysis using GSEABase [30] and Ingenuity Pathway Analysis (IPA) [31] were used to identify enriched gene sets and their roles in biological systems.

\section{Line $N$ transcriptional responses}

Despite the high levels of caecal $C$. jejuni M1 colonisation observed in susceptible line $\mathrm{N}$, only 8 and 3 DEGs were identified between infected and control birds at 1 and 5 dpi respectively (Table 1 ). At $1 \mathrm{dpi}$, all 8 DEGs were upregulated in infected compared to control birds whereas at $5 \mathrm{dpi}, 2$ DEGs were upregulated and 1 downregulated. DEGs relating to immune function included Interleukin 1 Receptor Like 1 (IL1RL1) and the C-C motif chemokine 7 (CCL7), which were both upregulated in infected compared to control line $\mathrm{N}$ birds at 1 and $5 \mathrm{dpi}$, respectively. Other DEGs detected in line $\mathrm{N}$ were involved in cell growth and survival such as Sestrin 2 (SESN2) and GTPase, IMAP Family Member 8 (GIMA $P 8)$, which were both upregulated in infected birds. Overall, RNA-Seq analysis revealed that $C$. jejuni colonisation in line $\mathrm{N}$ birds produced very limited changes in gene expression.

Due to the limited number of DEGs identified between control and infected line $\mathrm{N}$ birds, functional annotation analysis was performed on all 8 DEGs combined from both time points. GO term enrichment analysis did not identify any enriched gene sets in the caecal tonsils of line $\mathrm{N}$ birds following $C$. jejuni colonisation probably due to the limited number of DEGs. IPA identified molecular functions associated with the DEGs between infected and uninfected line $\mathrm{N}$ birds, with pathways involved in cell death and survival, cell to cell signalling

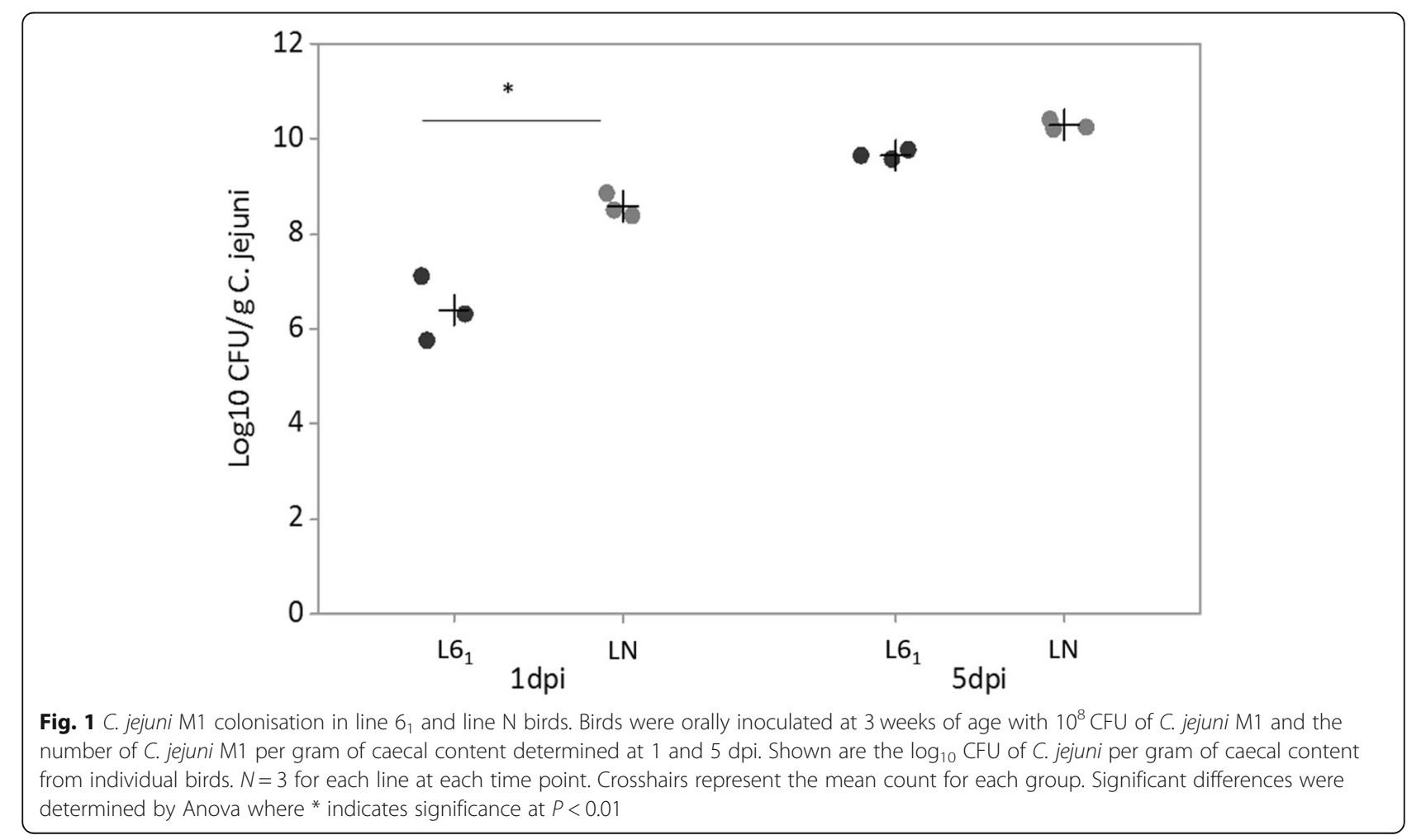


Table 1 DEGs between control and infected susceptible line $N$ birds at 1 and 5 dpi

\begin{tabular}{|c|c|c|c|c|c|}
\hline & Gene ID & Gene name & FC & $P$ Value & FDR \\
\hline \multirow[t]{8}{*}{ DE at $1 \mathrm{dpi}$} & ENSGALG00000005648 & SESN2 & 2.06 & $6.86 \mathrm{E}-07$ & $3.49 \mathrm{E}-03$ \\
\hline & ENSGALG00000041202 & FBXO32 & 1.98 & $4.56 \mathrm{E}-07$ & 3.49E-03 \\
\hline & ENSGALG00000016785 & IL1RL1 & 1.74 & $6.12 \mathrm{E}-06$ & $1.27 E-02$ \\
\hline & ENSGALG00000008885 & PDE1A & 1.52 & $8.40 \mathrm{E}-07$ & $3.49 \mathrm{E}-03$ \\
\hline & ENSGALG00000004058 & GPR146 & 1.51 & 2.07E-06 & $6.88 \mathrm{E}-03$ \\
\hline & ENSGALG00000008050 & HBP1 & 1.41 & $5.72 \mathrm{E}-07$ & $3.49 \mathrm{E}-03$ \\
\hline & ENSGALG00000008107 & IRS4 & 1.39 & $3.62 \mathrm{E}-06$ & $8.60 \mathrm{E}-03$ \\
\hline & ENSGALG00000013489 & CCDC82 & 1.37 & 3.33E-06 & $8.60 \mathrm{E}-03$ \\
\hline \multirow[t]{3}{*}{ DE at $5 \mathrm{dpi}$} & ENSGALG00000041079 & CCL7 & 9.58 & $3.19 \mathrm{E}-06$ & 2.23E-02 \\
\hline & ENSGALG00000044062 & GIMAP8 & 3.9 & $1.30 \mathrm{E}-06$ & $2.16 \mathrm{E}-02$ \\
\hline & ENSGALG00000031227 & ELP6 & 0.53 & 4.02E-06 & 2.23E-02 \\
\hline
\end{tabular}

and interaction and cellular function and maintenance being the most significant (Additional File 1: Fig. S1A). IPA also identified a significant network of genes involved in inflammatory responses (Additional File 1: Fig. S1B), indicating that $C$. jejuni may elicit a limited inflammatory response in susceptible line $\mathrm{N}$.

\section{Line $\boldsymbol{\sigma}_{1}$ transcriptional responses}

At 1 dpi, 69 DEGs were identified between infected and control line $6_{1}$ birds. Of these, 38 were upregulated and 31 were downregulated in $C$. jejuni-infected birds compared to controls (Additional file 2: Table S1). Genes involved in the activity of macrophages (including $M I P 1 a$ and MPEG1), natural killer (NK) cells and CD $8 \alpha^{+} \mathrm{T}$ lymphocytes (including EOMES, PRF1, CCL1, CD $8 \alpha$ chain-like 3 (ENSGALG00000032967), CD8 $\alpha$-like (ENSG $A L G 00000044720)$ ) were amongst those with the highest increase in expression in infected compared to control birds, indicating that early $C$. jejuni colonisation may stimulate inflammatory and/or antimicrobial responses in which these cell populations play a role.

Genes with the greatest reduction in expression following $C$. jejuni colonisation in line $6_{1}$ included members of the solute carrier family (SLC4A9, SLC26A4, $S L C 51 B), \mathrm{G}$ protein coupled receptor 6 member $\mathrm{A}$ (GPRC6A), TBC1 Domain Family Member 24 (TBC1D24), H6 Family Homeobox 2 (HMX2) and fibroblast growth factors (FGF19 and FGFBP1). At 5 dpi, no DEGs were identified between infected and uninfected line $6_{1}$ birds, despite the high levels of $C$. jejuni colonisation observed. None of the identified DEGs were shared between the two lines.

GO enrichment analysis of DEGs between infected and control line $6_{1}$ birds at 1 dpi identified 10 associated GO terms, seven of which were upregulated in infected birds. Immune-related GO terms associated with DEGs identified included 'Negative regulation of IL-17
Production', 'Chemokine Activity' and 'Interleukin 1 production', all of which were upregulated in response to $C$. jejuni colonisation (Additional File 3: Table S2). Of the three GO terms downregulated in response to $C$. jejuni colonisation, all were involved in nucleotide transport and processing.

By IPA 18 canonical pathways associated with DEGs were identified, of which 11 were immune-related (Fig. 2A). Some of the most significant of these included 'Communication between Innate and Adaptive Immune Cells', 'Phagosome Maturation', 'Granulocyte Adhesion and Diapedesis', 'Agranulocyte Adhesion and Diapedesis', 'TREM1 signaling' and 'Crosstalk between Dendritic Cells (DC) and Natural Killer Cells'. Other canonical pathways linked to resistance in line $6_{1}$ at 1 dpi included the FXR/RXR Activation and Iron Homeostasis signalling pathways. A number of molecular functions were identified as being significant to resistance in line $6_{1}$ birds following $C$. jejuni colonisation, the most significant including pathways concerning 'Molecular Transport', 'Lipid Metabolism' and 'Small Molecule Biochemistry' (Fig. 2B). Of the physiological functions found to be significantly related to $C$. jejuni resistance in line $6_{1}$, the most significant were related to immune function and included 'Hematological System Development and Function', 'Immune Cell Trafficking', 'Cell-Mediated Immune Responses', 'Lymphoid System Development and Function' and 'Hematopoesis' (Fig. 2C). IPA network analysis identified two significant networks of genes, involved in the antimicrobial response and cellular movement (Additional File 4: Fig. S2A) and lipid metabolism and transport (Additional File 4: Fig. S2B).

\section{Comparative analysis of responses between lines after $C$. jejuni infection}

To compare differences in pathway activation in response to $C$. jejuni colonisation between the two lines, 
(A) Canonical Pathways

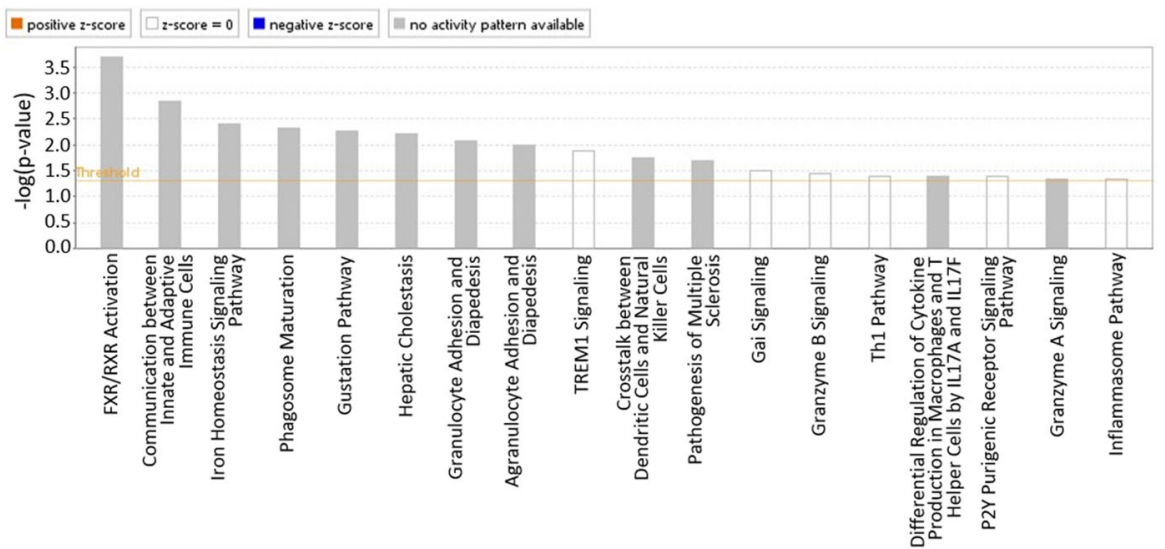

(B) Molecular functions

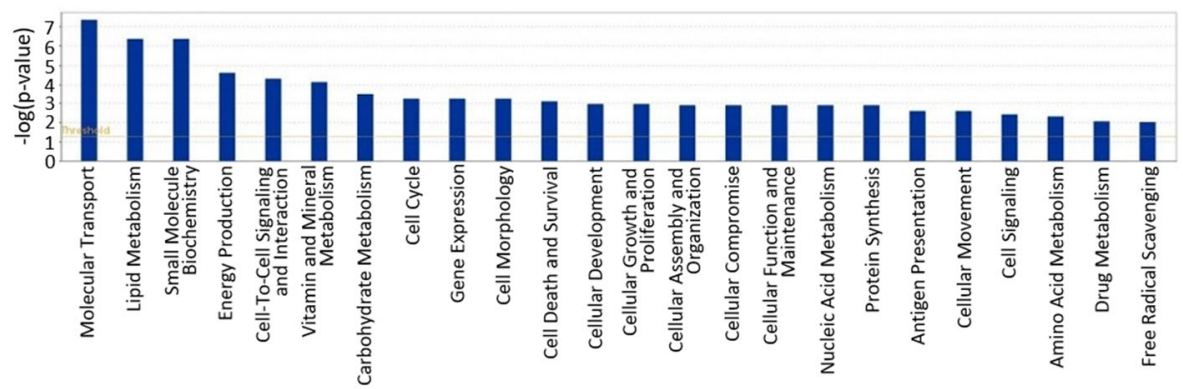

(C) Physiological functions

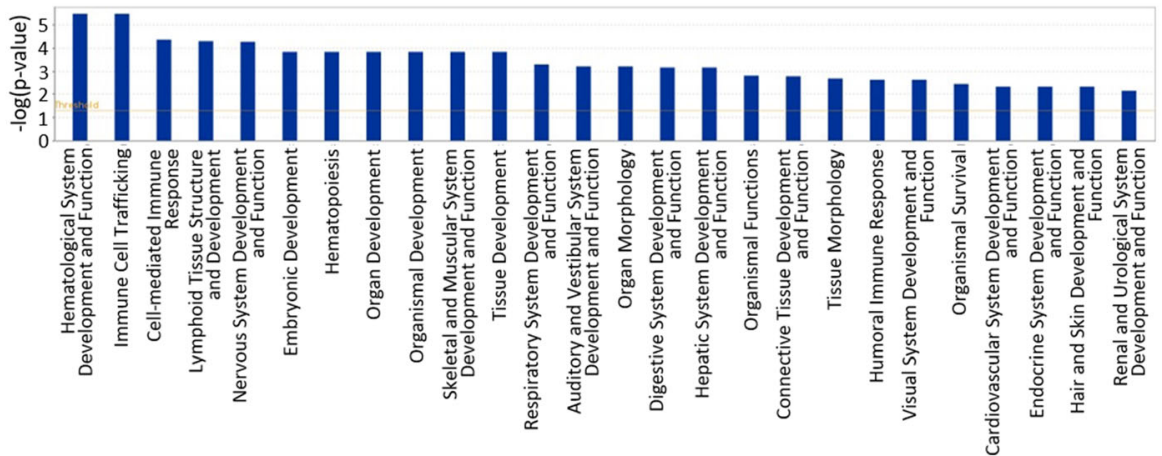

Fig. 2 IPA of DEGs in the caecal tonsils of control and C. jejuni M1 colonised line $\sigma_{1}$ birds at 1 dpi. Shown are the significant canonical pathways (A), molecular functions (B) and physiological functions (C) associated with DEGs. N=3 for both groups

an IPA comparison was performed between activated pathways in infected birds of each line at 1 dpi (Fig. 3). A number of immune-related pathways were found to be active in line $6_{1}$ birds at $1 \mathrm{dpi}$, but not in line $\mathrm{N}$ birds, including pathways involved in macrophage activity such as 'Phagosome Maturation', 'MIF-mediated Glucocorticoid', 'MIF Regulation of Innate Immune Responses', and the 'Inflammasome Pathway'. In contrast, pathways linked to Th2 ('IL-10 Signalling', the 'Th2 pathway') and
IL-6 responses ('STAT3 Pathway' and 'IL-6 Signalling') were activated in line $\mathrm{N}$ but not line $\sigma_{1}$ at $1 \mathrm{dpi}$ with $C$. jejuni. With few DEG identified in line $\mathrm{N}$, the same genes may underlie the pathways related to these responses. Pathways mainly involved in regulating bile and cholesterol in the liver, but which are also relevant to intestinal inflammation, were also activated to different extents in the caecal tonsils of the two lines at $1 \mathrm{dpi}$. These included the 'FXR/RXR Activation', 'Hepatic Cholestasis' 


\begin{tabular}{l} 
Canonical pathways \\
\hline Hepatic Cholestasis \\
FXR/RXR Activation \\
Gustation Pathway \\
Breast Cancer Regulation by Stathmin1 \\
Cardiac Hypertrophy Signaling (Enhanced) \\
Osteoarthritis Pathway \\
TREM1 Signaling \\
Toll-like Receptor Signaling \\
LXR/RXR Activation \\
Cellular Effects of Sildenafil (Viagra) \\
Hepatic Fibrosis / Hepatic Stellate Cell Activation \\
tRNA Splicing \\
Iron homeostasis signaling pathway \\
Phagosome Maturation \\
Acyl-CoA Hydrolysis \\
HOTAIR Regulatory Pathway \\
IL-10 Signaling \\
VDR/RXR Activation \\
Regulation of the Epithelial-Mesenchymal Transition Pathway \\
Inflammasome pathway \\
Nitric Oxide Signaling in the Cardiovascular System \\
PPAR Signaling \\
P38 MAPK Signaling \\
IL-6 Signaling \\
STAT3 Pathway \\
Th2 Pathway \\
Colorectal Cancer Metastasis Signaling \\
Cardiac $\beta$-adrenergic Signaling \\
Relaxin Signaling \\
MIF-mediated Glucocorticoid Regulation \\
Notch Signaling \\
Th1 and Th2 Activation Pathway \\
PI3K/AKT Signaling \\
Granulocyte Adhesion and Diapedesis \\
MIF Regulation of Innate Immunity \\
PPARa/RXRa Activation
\end{tabular}

Fig. 3 IPA comparison analysis of DEGs identified between line $\sigma_{1}$ and N C. jejuni M1 colonised birds at 1 dpi. Shown are significant canonical pathways identified from a comparison of DEGs between 3 infected compared to 3 control birds of each line. The degree of difference in expression is denoted by the depth of colour, with a darker colour indicating a greater degree of expression

and the 'Iron Homeostasis Signalling' pathways which were more active in line $6_{1}$ and the 'LXR/RXR activation', 'VDR/RXR activation' and 'PPAR signalling' pathways which were more active in line $\mathrm{N}$. These results indicate inherent differences in the regulation of immune pathways during the early stages of $C$. jejuni infection, which may have implications for $C$. jejuni colonisation of the caeca. Significant molecular functions were also associated with the DEGs between infected birds of the two lines, including those involved in lipid and amino acid metabolic pathways (Additional File 5: Fig. S3A). We also identified a significant network of genes, mainly expressed to a higher degree in line $\mathrm{N}$, relating to endocrine pathways (Additional File 5: Fig. S3B).

\section{Transcriptome comparison of uninfected line $\boldsymbol{\sigma}_{1}$ and line} $\mathrm{N}$ birds

\section{Gene expression}

To investigate inherent differences between chicken lines $6_{1}$ and $\mathrm{N}$, caecal tonsil transcriptomes were compared between control birds from each line. In total, 948 DEGs were identified between control birds of the two lines, pooled from both time points, of which 528 were more highly expressed in line $N$ compared to line $6_{1}$ (Additional File 6: Table S3). Genes with the highest level of expression in line $\mathrm{N}$ compared to line $6_{1}$ included Histone Cluster 1 H4 Family Member D (HIST1H4D), Ornithine Carbamoyltransferase (OTC), Choline O-Acetyltransferase (CHAT2), CD8 alpha 
chain-like (ENSGALG00000045876) and GTPase, IMAP Family Member 5-like (GIMAP5L). Several genes of the major histocompatibility complex I (MHCI) were also expressed to a greater extent in line $\mathrm{N}$, including $\mathrm{MHCI}$ $A 1, M H C B L 2$ and $M H C I Y$. Mucin 2 (MUC2), $\beta$-defensin $10(A v B D 10)$ and granzyme A (GZMA) were also expressed at a significantly higher level in susceptible line N. Interestingly, two genes identified in the QTL regions associated with $C$. jejuni colonisation in these lines were expressed at higher levels in line N. Acid Sensing Ion Channel Subunit Family Member 4 (ASIC4) was present in the QTL region on Chromosome 7 whereas ENSGALG00000028367, a zinc finger protein, was in the QTL identified on Chromosome 16 [21].

Of the DEGs identified between line $6_{1}$ and $N$ birds, 420 genes were expressed at higher levels in line $6_{1}$ compared to line N. Of these, those with the greatest foldchange in expression included Class I histocompatibility antigen, F10 alpha chain-like (LOC107050538), Forkhead Box M1 (FOXM1), adenylate cyclase 5 (ADCY5), Deleted In Malignant Brain Tumors 1 (DMTB1), BPI Fold Containing Family B Member 3 (BPIFB3). Several other genes more highly expressed in line $6_{1}$ included the macrophage marker CD163-like protein (DMBT1L), glutathione peroxidase 2 (GPX2; involved in protection against oxidative stress), and trefoil factor 2 (TFF2; involved in stabilisation of the mucosal layer and healing of the epithelial layer).

\section{Functional analysis}

GO enrichment analysis performed on DEGs between the control birds of each line identified 10 associated GO terms, five of which were enriched in each line and some of which had immune function (Additional File 7: Table S4). Immune-related GO terms enriched in line $6_{1}$ compared to line $\mathrm{N}$ included the 'Detection of Molecules of Bacterial Origin', 'Negative Regulation of IL- $1 \beta$ Production' and 'Negative Regulation of Hematopoietic Progenitor Cell Differentiation' whereas GO terms enriched in line $\mathrm{N}$ compared to line $6_{1}$ included 'Negative Regulation of Viral Release from Host Cell' and 'Negative Regulation of Leukocyte Chemotaxis', indicating that these chicken lines may be in different states of immune readiness prior to their interactions with pathogens.

IPA further identified inherent differences in the level of activity of canonical pathways between the two lines (Fig. 4A). Blood coagulation pathways were more activated in line N, and included the 'Coagulation System' and 'Intrinsic Prothrombin Pathway'. The 'eNOS signalling' pathway was also more activated in line N. Pathways more active in resistant line $6_{1}$ included 'Estrogen Biosynthesis' and 'Nicotine Degradation II and III'. IPA also identified significant differences in molecular functions, with the most significant being 'Cell-to-Cell Signalling and Interaction', 'Molecular Transport' and 'Protein Synthesis' (Fig. 4B).

Significant networks of genes associated with cell-tocell signalling (Additional File 8: Fig. S4A), gastrointestinal pathways (Additional File 8: Fig. S4B) and amino acid (Additional File 8: Fig. S4C) and lipid metabolism (Additional File 8: Fig. S4D) were identified with higher activity in line $\mathrm{N}$ compared to line $6_{1}$, highlighting that these two lines may be in different metabolic states prior to $C$. jejuni challenge and susceptibility to $C$. jejuni in line $\mathrm{N}$ may be due in part to distinct metabolism. Furthermore, some genes potentially acting as upstream regulators of DEGs were found to be significantly upregulated in line $\mathrm{N}$, including the $\mathrm{B}$-cell receptor (BCR) (Additional File 9: Fig. S5A), microRNA mir155 (Additional File 9: Fig. S5B) and the nuclear factor of activated T-cells (NFAT) (Additional File 9: Fig. S5C).

\section{Gene cluster analysis}

Graphia software [32] analysis revealed the most prominent clustering was by bird line, suggesting that basal gene expression differences between lines $6_{1}$ and $\mathrm{N}$ may explain intrinsic resistance as opposed to differences in their response to $C$. jejuni infection. Two components containing the majority of DEGs were identified. These were Component 1 comprising of 2822 genes expressed to a greater extent in line $\mathrm{N}$ and Component 2 comprising of 2285 genes expressed to a greater extent in line $6_{1}$ (Fig. 5A and B respectively). Mean histogram plots of all genes present within these two components indicated that genes were generally expressed at higher levels in one line compared to the other indicating major differences in the regulation of groups of genes are key to the resistance and susceptible phenotypes in these lines (Fig. $5 \mathrm{C}$ and $\mathrm{D})$.

\section{Validation of DEGs by qRT-PCR}

RNA-Seq data was validated by qRT-PCR analysis of a subset of genes. These were chosen for validation based on their possible biological significance during $C$. jejuni colonisation and the degree to which they were DE. Genes were mainly selected from the pairwise comparison between control birds of each line, owing to the high number of DEGs identified in this group. Correlation of the qRT-PCR results with the RNA-Seq results produced a correlation co-efficient of $\mathrm{R}^{2}=0.86(p<0.001)$ therefore the qRT-PCR results are comparable to the RNASeq data (Fig. 6).

\section{Discussion}

With the handling or consumption of contaminated chicken accounting for a high proportion of human campylobacteriosis $[6,7]$, a pressing need exists to 
(A) Canonical pathways

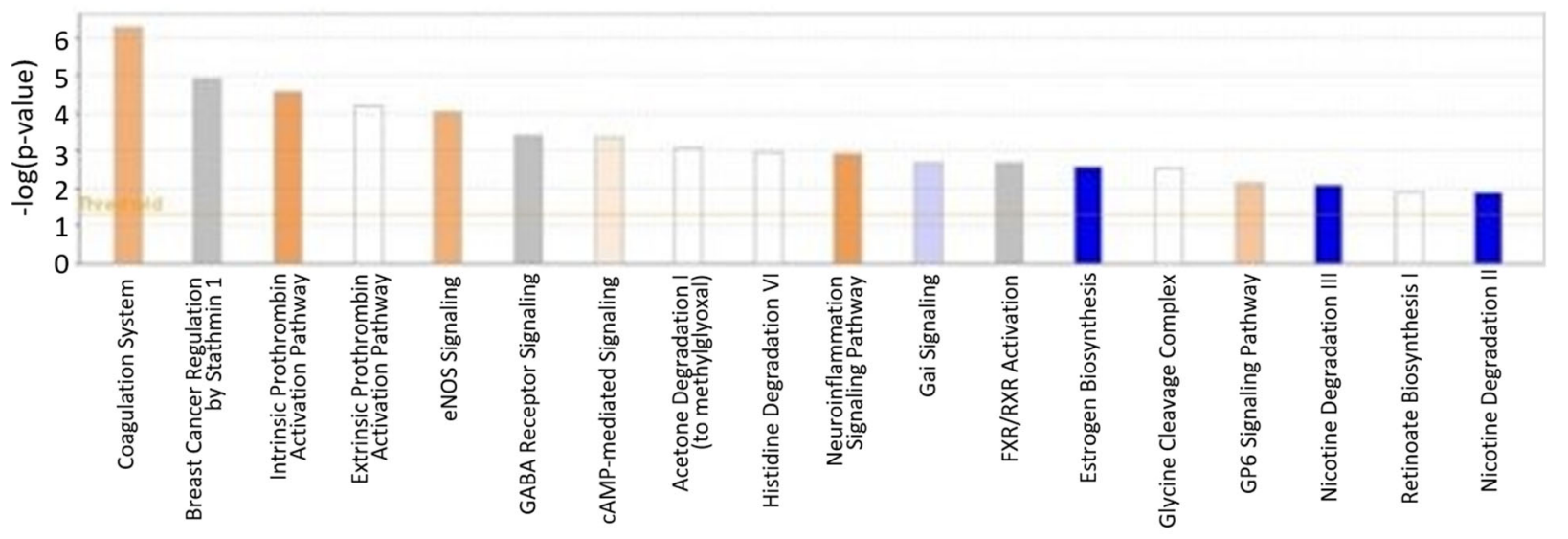

(B) Molecular functions

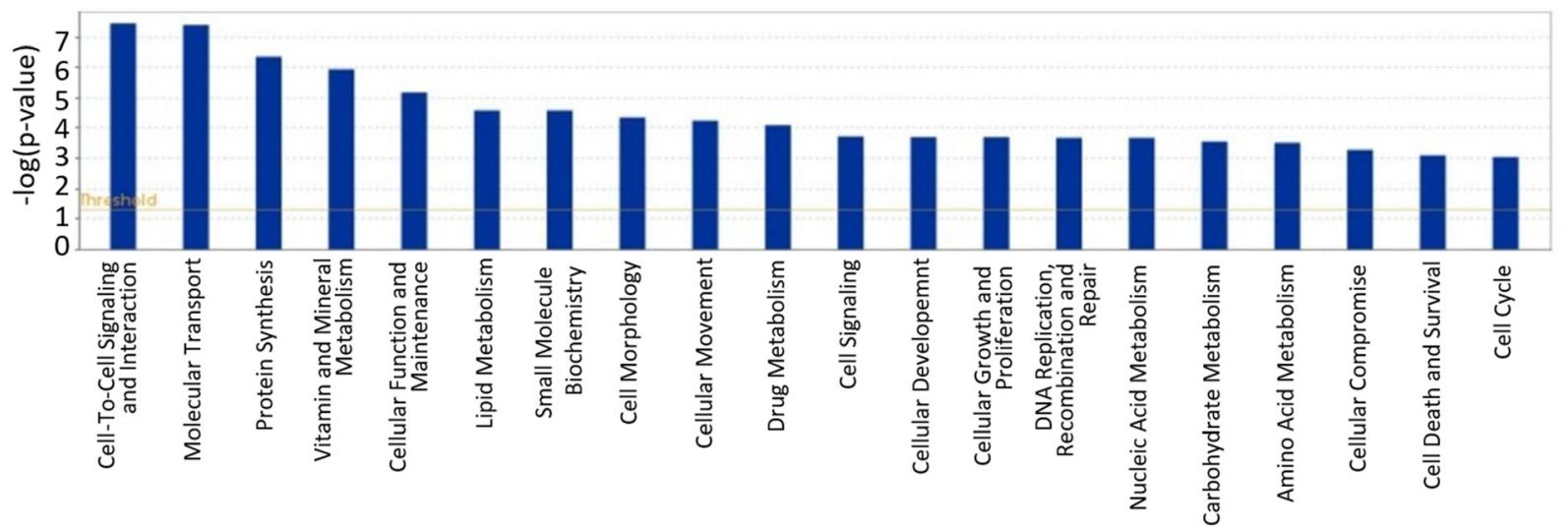

Fig. 4 IPA of DEGs identified between control birds of line $\sigma_{1}$ and N. Shown are significant canonical pathways (A) and molecular functions (B) associated with DEGs between control birds. In $(\mathbf{A})$, red bars indicate canonical pathways upregulated in line $\mathrm{N}$ and blue bars indicate pathways upregulated in line $\sigma_{1} . N=6$ for each line (3 control birds pooled from each time point)

reduce the prevalence of $C$. jejuni in commercial broiler flocks. White Leghorn inbred lines $6_{1}$ and $N$ have an established difference in heritable resistance to $C$. jejuni colonisation, with genetic variation associated with $C$. jejuni resistance identified by genome-wide association studies using backcross and advanced $6_{1} \times \mathrm{N}$ intercross populations [21]. Furthermore, key QTL associated with resistance to $C$. jejuni are shared between these inbred lines and commercial broilers [14] indicating that findings in inbred chickens can be highly relevant to $C$. jejuni control in commercial flocks. Using RNA-Seq, we evaluated transcriptomic differences in the caecal tonsils of these inbred lines, both inherently and following $C$. jejuni colonisation, to investigate the basis of their differential resistance to $C$. jejuni and potentially obtain biomarkers that could be selected in commercial populations.

We showed that compared to line $\mathrm{N}$, line $6_{1}$ was relatively resistant to early $C$. jejuni M1 colonisation in the caeca at $1 \mathrm{dpi}$, however by $5 \mathrm{dpi}$ both lines were similarly susceptible to colonisation. Previously, Boyd et al reported resistance to cloacal and caecal colonisation was apparent in line $6_{1}$ compared to line $\mathrm{N}$ from 4 to $20 \mathrm{dpi}$ [20], a discrepancy with this study likely due, in part, to the different $C$. jejuni strains used by Boyd ( $C$. jejuni $14 \mathrm{~N}$ and 81-176) and in this report (C. jejuni M1). Chintoan-Uta et al recently reported line $\mathrm{N}$ birds to be colonised by C. jejuni $11168 \mathrm{H}$ at c. $10^{4} \mathrm{CFU} / \mathrm{g}$ caecal contents 9 dpi at 3 weeks-of-age whereas the challenge strain was absent at the limit of detection by direct plating at this time in line $6_{1}$ birds [29]. M1 rapidly colonises the chicken caeca from doses as low as $100 \mathrm{CFU}$ $[33,34]$ and both the rate of colonisation and caecal burden have proven greater than for other $C$. jejuni strains tested in the same model, including $11168 \mathrm{H}$ (Stevens et al, unpublished data). For many $C$. jejuni strains, there is a minimum infective dose which can cause high levels of colonisation in the chicken caeca [35-37]. It is plausible that both chicken lines may have been overwhelmed by the dose of $C$. jejuni M1 administered, now known to 


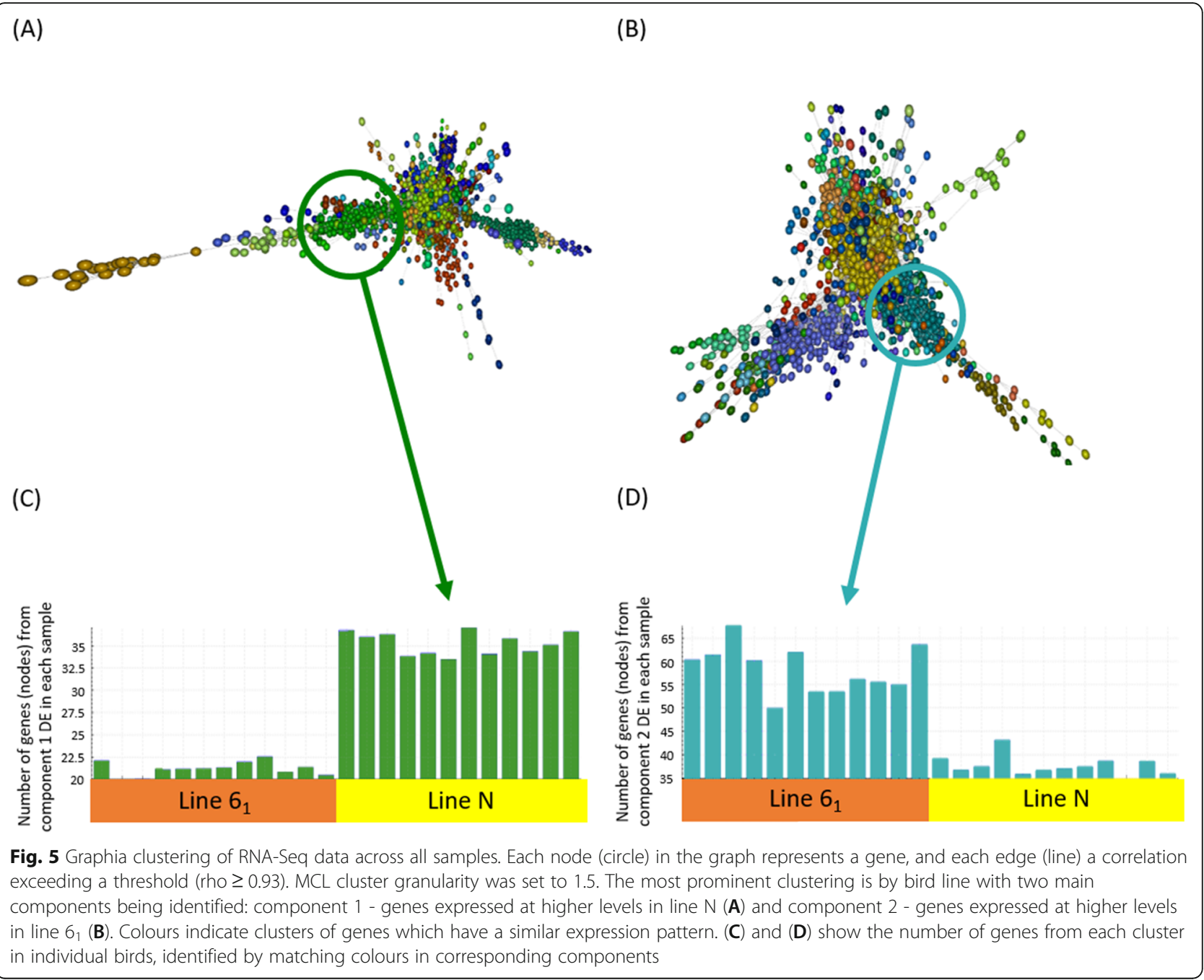

be considerably higher than the minimum dose required for reliable colonisation $\left(10^{2} \mathrm{CFU},[33]\right)$.

Susceptible line $\mathrm{N}$ displayed a very limited caecal transcriptional response to $C$. jejuni colonisation compared to resistant line $6_{1}$ where a wider, albeit brief, response at $1 \mathrm{dpi}$ was observed. Although functional annotation analysis identified significant pathways related to immune function in both lines, the difference in magnitude between the responses of the two lines may partly explain the relative resistance and susceptibility displayed at 1 dpi. DEGs related to macrophage, NK cell and $\mathrm{CD} 8 \alpha^{+} \mathrm{T}$ cell activity were upregulated in resistant line $6_{1}$ at $1 \mathrm{dpi}$, with functional annotation analysis identifying pathways involved in communication between the innate and adaptive arms of immunity, phagosome maturation and crosstalk between NK cells and DC as involved. It follows that the lower level of colonisation in line $6_{1}$ at $1 \mathrm{dpi}$ may, in part, be attributable to this innate response whereas at $5 \mathrm{dpi}$, this response is absent and coincides with line $6_{1}$ caecal colonisation levels matching those of line N. It is possible that NK cells are involved in the initial response to $C$. jejuni observed in line $6_{1}$ as the NK complex is located in close proximity to the chicken MHC B complex, which influences the responsiveness of chicken NK cells [38], and several DE MHCI genes were identified between lines $\mathrm{N}$ and $6_{1}$, including MHCIA1, MHCBL2 and MHCIY.

Innate inflammatory responses have been linked to reduced $C$. jejuni colonisation elsewhere. Although not detected in our study, Psifidi et al found CXCLi1 and CXCLi2 (proinflammatory chemokines involved in heterophil chemotaxis) expression was reduced in the caecal tonsils of both line $6_{1}$ and $\mathrm{N}$ following $C$. jejuni infection [21], but more so in line $\mathrm{N}$, further implying innate responses may be involved in controlling $C$. jejuni colonisation at this site. Moreover, broilers selected for an inflammatory phenotype (high levels of IL-6, CXCLi2 and CCLi2) are less likely to become colonised by $C$. 


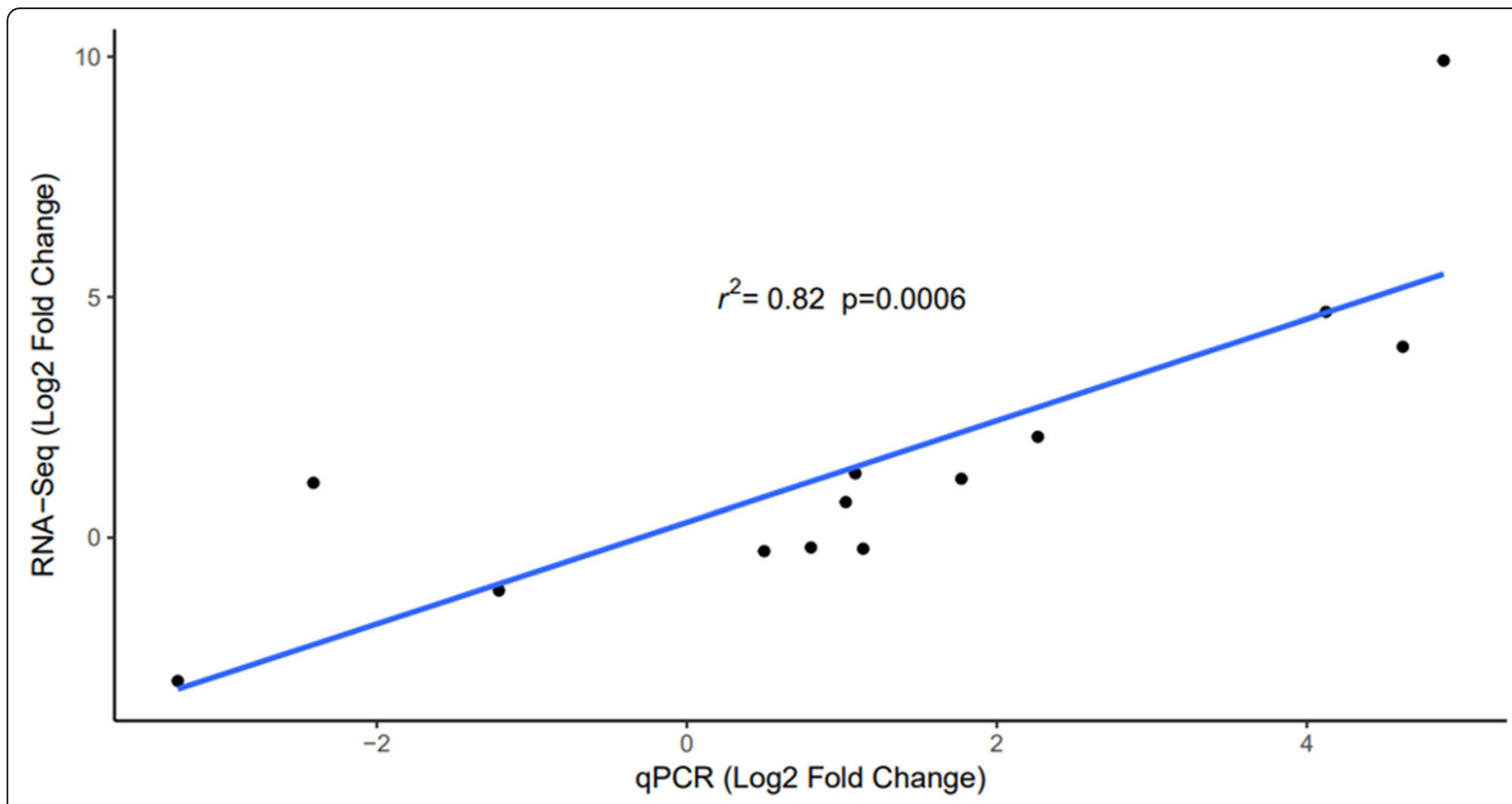

Fig. 6 qRT-PCR validation of DEGs identified by RNA-Seq. Shown is the correlation of the $\log _{2}$ fold change in mRNA transcripts as determined by RNA-Seq (y-axis) with the $\log _{2}$ fold change of mRNA transcripts as determined by qRT-PCR ( $x$-axis). DEGs that were validated are given in Additional file 11: Table 6 . The line of best fit is represented in blue with the $R^{2}$ value denoted on the graph

jejuni compared to those selected for lower inflammatory phenotype [22], indicating that an inherent proinflammatory status reduces the ability of $C$. jejuni to colonise the chicken caeca. However, in contrast, Humphrey et al found that levels of CXCLi1 and CXCLi2 expression were not related to differences in caecal $C$. jejuni load [27].

Iron homeostasis signalling pathways were upregulated in resistant line $6_{1}$ at $1 \mathrm{dpi}$. Iron is essential for bacterial replication and mutants with defects in iron acquisition are frequently attenuated [39], therefore differences in iron availability in the gastrointestinal tract between chicken lines may factor in their relative resistance and merits further investigation. Additionally, higher activity related to MIF-mediated glucocorticoid, the MIF innate immune response and inflammasome pathways occurred during initial C. jejuni colonisation in line $6_{1}$, indicating that MIF and the inflammasome may be mediators in the initial inflammatory response in line $6_{1}$.

Major transcriptional differences were apparent between control birds of both lines, and were far greater than those observed in response to $C$. jejuni infection. Our study is not the first to identify such a degree of inherent transcriptional variation between White Leghorn inbred lines. For example, gene expression in the spleen and thymus differs by several hundred genes between lines $6_{1}$ and $7_{2}$, which are relatively resistant and susceptible to Marek's Disease Virus respectively [40].
Significant networks of genes associated with amino acid and lipid metabolism were upregulated in line $\mathrm{N}$ control birds, consistent with other studies linking higher inherent metabolic states with susceptibility to C. jejuni. Li et al (2010) found amino acid, lipid and glucose metabolic pathways to be upregulated in a $C$. jejuni-susceptible line compared to a resistant line following colonisation, with amino acid processes also upregulated in control birds of the susceptible line [15]. Additionally, Li et al (2011) found increased expression of genes involved in fatty acid and protein metabolic processes between C. jejuni-colonised compared to non-colonised birds of the same line [16]. Our data identified differences in transcription of genes associated with metabolic activity between the lines coinciding with differential colonisation levels at 1 dpi. For example, higher activation in the farnesoid $x$ receptor (FXR)/retinoid $x$ receptor activation was present in line $6_{1}$ but liver $\mathrm{x}$ receptor (LXR)/RXR, vitamin D receptor (VDR)/RXR and peroxisome proliferator-activated receptor $(\mathrm{PPAR} \alpha) / \mathrm{RXR}$ activation was higher in line N. FXR, LXR and PPAR- $\alpha$ are mainly associated with lipid metabolism and in the regulation of triglyceride levels in mammals [41-43]. However, between them, FXR, LXR and PPAR- $\alpha$ also have various roles in regulating intestinal inflammation and immunity including effects on macrophage inflammatory activity, reducing the presence of reactive oxygen species (ROS) and maintaining the intestinal barrier [44-49], 
and are considered potential therapeutic targets in the case of mammalian IBD [45, 50, 51]. Expression of FXR, LXR and PPAR- $\alpha$ in these chicken lines may therefore modulate intestinal inflammatory responses, influencing C. jejuni colonisation. Furthermore, their roles in triglyceride metabolism may also influence colonisation as triglyceride-rich lipoprotein can bind LPS, reducing LPS toxicity during bacterial infection and reducing macrophage activation [52]. Vitamin D and its receptor are important in maintaining the mucosal barrier of the intestine [53] and modulate proinflammatory cytokine production [54]. Abiotic $I L 10^{-/-}$mice treated with artificial vitamin $\mathrm{D}$ suffered less diarrhoea and had lower levels of intestinal IL-6, IFN- $\gamma$ and CCL2 during campylobacteriosis than mice that did not receive vitamin $\mathrm{D}$ [55], therefore the contribution of vitamin D and the vitamin $\mathrm{D}$ receptor to $C$. jejuni susceptibility in line $\mathrm{N}$ chickens warrants further study. Upstream gene regulators involved in several immune-related networks were associated with the DEGs identified between control birds of both lines (BCR, NFAT and miR-155). Previously, increased caecal miR-155, a regulator of inflammatory processes in mammals [56], was observed following C. jejuni colonisation in chickens [57]. These differences in inflammatory states prior to $C$. jejuni inoculation likely influence the speed and resulting level of colonisation in different chicken lines.

Lines $6_{1}$ is also relatively resistant to $S$. Typhimurium colonisation compared to line $\mathrm{N}[28,58]$. QTLs associated with resistance to both $S$. Typhimurium and $C$. jejuni have been identified on chromosome 14 [21, 59] and chromosome 16 [21, 60,61] implying that similar mechanisms of genetic control may confer resistance to both C. jejuni and Salmonella. Furthermore, greater inflammatory responses to Salmonella have been observed in resistant chicken lines compared to susceptible birds [62] highlighting the importance of innate responses in resistance to enteric bacterial pathogens.

As C. jejuni mainly resides in the chicken intestinal lumen, it is plausible that differences at the mucosal surface or extracellular milieu may account for resistance. Genes encoding MUC2 and AvBD10 were expressed to a higher level in line $\mathrm{N}$ compared to line $6_{1}$ control birds. Previously, it has been shown that chicken mucus inhibits $C$. jejuni invasion of both chicken and human primary intestinal cells compared with human mucus [63]. It is therefore possible that inherent differences in the composition of chicken mucus between lines accounts for the differences in the colonisation levels observed. GZMA was also more highly expressed in line $\mathrm{N}$, corresponding to a microarray study where GZMA expression was higher in the caeca of $C$. jejunicolonised compared to non-colonised birds, indicating GZMA is linked with elevated C. jejuni levels in the caeca [16]. Recent studies have identified mostly limited differences in the microbial communities present in the caeca of lines $6_{1}$ and $\mathrm{N}$ at the age they were inoculated herein, but the extent to which these differences drive distinct patterns of gene expression in the gut, or vice versa, is unknown [29].

Other studies have examined the impact of gene expression and host genetics on $C$. jejuni colonisation of the caeca $[14,16,17,19,20,23,24]$. However, these varied in the strain of $C$. jejuni used, bird line and challenge age of the birds (between 2 and 4 weeks-of-age), and sampling intervals (from $6 \mathrm{~h}$ to $14 \mathrm{dpi}$ ) resulting in variation of the level of colonisation observed and challenges in extrapolating from one study to the next. Despite these differences, a similar narrative can be seen throughout these studies whereby a moderate number of significant DEGs was identified upon Campylobacter challenge, but often at a low fold-change indicating that even high levels of $C$. jejuni colonisation does not elicit a dramatic response in the chicken $[16,17,21,64]$. We elected to study the transcriptome of caecal tonsils as a gut-associated lymphoid tissue at a key site of Campylobacter persistence, and to permit direct comparison with RNA-Seq data from resistant or susceptible broilers from a recent genome-wide association study [14] and earlier qRT-PCR data on candidate resistance-associated genes in lines $6_{1}$ and $N$ [21]. The former study detected differentially transcribed genes in caecal tonsils within quantitative-trait loci associated with resistance to caecal colonisation, indicating that it is a relevant anatomical location to sample. However, we cannot preclude the possibility that the responses observed may not be typical of the wider caecal mucosa, or tissue at other key sites of Campylobacter persistence in poultry.

A GWAS on an advanced intercross of line $6_{1}$ and N previously identified 6 SNPs on chromosomes 4, 14 and 16 associated with resistance to $C$. jejuni colonisation [21]. We identified two genes located within the QTL regions associated with $C$. jejuni colonisation in these lines, ASIC4 (Acid Sensing Ion Channel Subunit Family Member 4) and ENSGALG00000028367, both of which were inherently expressed to a greater extent in line $\mathrm{N}$. ASIC4 is broadly expressed in the mammalian nervous system but its role in birds is unknown. In mammals, ASICs are known for their role in neuroinflammation [65] and promote exocytosis and maturation in bone marrow-derived macrophages stimulated by extracellular acidosis [66]. ENSGALG00000028367, or BZFP2, is a zinc finger-like protein which likely binds nucleic acids, proteins and other small molecules [67], and bares similarity to the CKR1-like gene and is present in a region of the MHC-B locus [68].

To conclude, RNA-Seq analysis demonstrated an initial inflammatory response to $C$. jejuni infection of a 
greater magnitude in resistant line $6_{1}$ compared to line $\mathrm{N}$, which may be associated with caecal colonisation. This response was short lived and absent at later intervals when differential resistance was not detected. By far, the most striking differences in gene expression were detected between uninfected control birds of the two lines. The identification of candidate genes involved in early innate responses and metabolic pathways provides a foundation for future studies on avian heritable resistance to Campylobacter.

\section{Methods}

\section{Animals}

Two inbred White Leghorn chicken lines were used in this study. Lines $6_{1}$ and $N$, reported to be relatively resistant and susceptible to $C$. jejuni colonisation respectively, originate from the USDA ARS Avian Disease and Oncology Laboratory (formerly the Regional Poultry Laboratory) in East Lansing, MI, USA [12]. Flocks of both lines were maintained under specified pathogen-free (SPF) conditions at the National Avian Research Facility (NARF) at the Roslin Institute, UK.

\section{Bacterial culture, experimental design and sample collection}

C. jejuni strain M1 [69] was used to inoculate animals in this study. C. jejuni M1 was cultured on modified charcoal-cephoperazone-microaerophillic agar (mCCDA; Oxoid) or in Mueller-Hinton (MH) broth (Oxoid) at $37{ }^{\circ} \mathrm{C}$ with $5 \% \mathrm{O}_{2}, 5 \% \mathrm{CO}_{2}$ and $90 \% \mathrm{~N}_{2}$ in a microaerophilic cabinet. Liquid cultures in broth were with shaking at $400 \mathrm{rpm}$ under the same atmospheric conditions.

Twelve birds each of White Leghorn lines $6_{1}$ and $\mathrm{N}$ were obtained on the day of hatch and housed under SPF conditions with access to feed and water ad libitum. For each line there were 6 control and 6 infected birds. At 3 weeks-of-age, birds of the infected groups were challenged by oral gavage with $10^{8}$ C. jejuni $\mathrm{M} 1$ in $0.1 \mathrm{ml}$ of $\mathrm{MH}$ broth and birds of the control groups given an equivalent volume of MH broth only. At 1 and 5 dpi, 3 chickens of each group were culled by cervical dislocation and death confirmed by permanent cessation of blood circulation. At post mortem examination, caeca were collected for the enumeration of $C$. jejuni by plating $100 \mu \mathrm{l}$ of triplicate 10 fold serial dilutions of caecal contents in phosphatebuffered saline (PBS) to mCCDA plates. Caecal tonsils were collected promptly into RNAlater ${ }^{\circ}$ (Life Technologies) and stored at $-80^{\circ} \mathrm{C}$ until processing.

\section{RNA-Seq library preparation, sequencing and data analysis}

RNA was extracted from both caecal tonsils using the Qiagen RNeasy mini kit according to the manufacturer's instructions. RNA preparations were assessed for quantity and quality using a Qubit ${ }^{\mathrm{Tm}}$ Fluorimeter (Invitrogen) and an Agilent 2200 TapeStation (Agilent Technologies) respectively. The resulting RNA integrity number (RIN) values for all RNA samples was greater than 9 therefore all 24 samples $(n=3$ per group) were subsequently submitted to the Edinburgh Genomics sequencing facility (Edinburgh, UK) for RNA-Seq. The TruSeq stranded mRNA-Seq Library Prep kit (Illumina) was used to generate mRNA libraries free from ribosomal RNA and the resulting transcriptomes sequenced using the Illumina HiSeq 4000 system to generate $75 \mathrm{bp}$ paired end reads at a depth of 50 million reads per sample.

The quality of the raw sequence reads was assessed using FASTQC (v0.11.5) [70]. Cutadapt (v1.14) [71] was used to trim adapter sequences, remove bases with a Phred score of less than 30 and ensure resulting sequences were at least $50 \mathrm{bp}$ in length. Trimmed reads were then mapped to the Gallus gallus reference genome (GalGal5, Ensembl release 89) using the STAR aligner software package (v2.5.1b) [72] with a minimum alignment of 52.5 million input reads for each sample (Additional File 10: Table S5). In all samples, over 90\% of reads were uniquely mapped with fewer than $10 \%$ either unmapped or mapped to multiple loci. The number of reads aligning to genomic features were determined using FeatureCounts (v1.5.3) [73] using default parameters. Analysis of DEGs was performed in R using EdgeR package with DEGs exhibiting a fold change $(\mathrm{FC})>2$ and a false discovery rate $(\mathrm{FDR})<0.05$ considered significant.

Gene ontology term enrichment analysis was performed in $\mathrm{R}$ using the GSEABase package (downloaded from the Gene Set Enrichment Analysis (GSEA) website, https://software.broadinstitute.org/ gsea/index.jsp, file: C5.all.v6.0.symbols). Significant gene ontology was determined using the mRoast function of the Limma package in $\mathrm{R}$ for each pairwise comparison. Ingenuity Pathway Analysis (IPA) was performed on significant DEGs using IPA software (Qiagen Bioinformatics, www.qiagenbioinformatics. com/products/ingenuity-pathway-analysis) to classify their associated biological functions, canonical pathways and biological networks. Network analysis was performed using the normalised raw counts in Graphia Pro [32] with a Pearson correlation threshold of $\mathrm{r}=0.93$. The number of nodes (genes) in the analysis was 6066 linked by $181.1 \mathrm{k}$ edges. Markov Clustering (MCL) was performed with a granularity of 1.5 on these networks to identify components containing genes of similar expression patterns. Components of less than 10 nodes were removed from the analysis. Gene lists derived from Graphia were submitted to the functional annotation tool DAVID [74] to further 
investigate the roles of genes identified in the components, with Gallus gallus selected as the background for these lists.

\section{cDNA preparation and quantitative reverse-transcriptase polymerase chain reaction (qRT-PCR)}

Genes related to immune function that were observed to be DE by RNA-Seq were validated by qRT-PCR. The Verso cDNA Synthesis kit (Thermo Scientific) was used to prepare cDNA from $1 \mu \mathrm{g}$ of total RNA according to the manufacturer's instructions. Quantitative PCR reactions were performed using the Forget-Me-Not ${ }^{\text {tw }}$ qPCR Master Mix (Biotium) in $20 \mu \mathrm{L}$ volumes containing $1 \mathrm{X}$ Forget-Me- Not $^{\mathrm{Tw}}$ qPCR Master Mix, $0.5 \mu \mathrm{M}$ each of the forward and reverse primers, $50 \mathrm{nM}$ of ROX reference dye and $2 \mu \mathrm{L}$ of cDNA at a 1:10 dilution in template buffer. Gene-specific primers were purchased from Sigma and are detailed in Additional File 11: Table S6. The amplification and detection of specific DNA was achieved using the AB 7500 FAST Real-Time PCR System (Applied Biosystems) to the following program: $95^{\circ} \mathrm{C}$ for $2 \mathrm{~min}$ followed by 40 cycles of $95^{\circ} \mathrm{C}$ for $5 \mathrm{~s}$ then $60^{\circ} \mathrm{C}$ for $30 \mathrm{~s}$. To confirm the presence of a single PCR product, melting curves were generated by 1 cycle of $60^{\circ} \mathrm{C}$ for $1 \mathrm{~min}$, increasing to $95^{\circ} \mathrm{C}$ in $1 \%$ increments every $15 \mathrm{~s}$. Samples were run in triplicate and each qPCR experiment contained 3 no-template controls and a 5fold dilution series in duplicate of pooled caecal tonsilderived cDNA from several birds from which standard curves were generated. The relative expression of genes were calculated as described by Pfaffl [75] and normalised to the geometric mean of three reference genes; r28S, TBP and GAPDH. A Spearman's correlation was calculated between the $\log _{2}$ fold-change of the RNA-Seq results to the $\log _{2}$ fold-change detected by qPCR in $\mathrm{R}$.

\begin{abstract}
Abbreviations
cDNA: Complementary DNA; DC: Dendritic cell; DE: Differentially expressed; DEGs: Differentially expressed genes; dpi: Days post-infection; FC: Fold change; FDR: False discovery rate; GO: Gene ontology; IPA: Ingenuity Pathway Analysis; mCCDA: Modified charcoal-cefoperazone-deoxycholate agar; NK: Natural killer; qRT-PCR: Quantitative reverse-transcriptase polymerase chain reaction; SPF: Specified pathogen-free
\end{abstract}

\section{Supplementary Information}

The online version contains supplementary material available at https://doi. org/10.1186/s12864-021-07748-2.

Additional file 1: Figure S1. IPA of DEGs in the caecal tonsils between control and C. jejuni M1 colonised line $\mathrm{N}$ birds at $1 \mathrm{dpi}$. Shown are significant molecular functions (A) associated with DEGs and a significant network of inflammatory responses involved during $C$. jejuni infection of line $\mathrm{N}$ at $1 \mathrm{dpi}(\mathrm{B})$. In (B), genes or nodes coloured red are upregulated in C. jejuni colonised birds whereas those in green are downregulated. $N=3$ for both groups.

Additional file 2: Table S1. DEGs in the caecal tonsils between control and infected line $\sigma_{1}$ birds at $1 \mathrm{dpi}$. A fold change greater than 1 indicates genes that were upregulated in infected compared to control birds whereas a fold change less than 1 indicates genes which were downregulated in infected compared to control birds. $N=3$ for both groups. FC: fold change, FDR: False Discovery Rate.

Additional file 3: Table S2. Significant GO Terms associated with DEGs in the caecal tonsils between infected and control line $\sigma_{1}$ birds at $1 \mathrm{dpi}$. DEGs were identified between 3 infected and 3 control birds. FDR: False Discovery Rate.

Additional file 4: Figure S2. IPA of DEGs in the caecal tonsils of control and C. jejuni M1 colonised line $\sigma_{1}$ birds at $1 \mathrm{dpi}$. Shown are a significant network of genes involved in the antimicrobial response and cellular movement (A) and of genes involved in lipid metabolism and transport (B). Genes or nodes coloured red are upregulated in colonised compared to control birds whereas those in green are downregulated.

Additional file 5: Figure S3. IPA comparison of DEGs identified in the caecal tonsils between line $\sigma_{1}$ and $\mathrm{N}$ C. jejuni M1 colonised birds at $1 \mathrm{dpi}$. Shown are significant molecular functions (A) and a significant network of genes related to endocrine pathways (B) identified from the comparison of DEGs between 3 infected and 6 control birds of each line. In (B), genes or nodes coloured red are upregulated in colonised line $N$ birds whereas those in green are upregulated in colonised line $\sigma_{1}$ birds.

Additional file 6: Table S3. DEGs in the caecal tonsils between line N and line $\sigma_{1}$ control birds. Analysis compares the gene expression of all six control birds for each line. A fold change greater than 1 indicates genes more highly expressed in line $\mathrm{N}$ whereas a fold change less than 1 indicates genes more highly expressed in line $6_{1}$. FC: fold change, FDR: False Discovery Rate.

Additional file 7: Table S4. Significant GO Terms associated with DEGs in the caecal tonsils between line $\mathrm{N}$ and line $\sigma_{1}$ control birds. Upregulated $\mathrm{GO}$ terms are associated with genes with higher expression in line $\mathrm{N}$ and downregulated $\mathrm{GO}$ terms are associated with genes with higher expression in line $6{ }_{1}$. DEGs were identified between all six control birds of each line. FDR: False Discovery Rate.

Additional file 8: Figure S4. IPA of DEGs in the caecal tonsils identified between control birds of line $\sigma_{1}$ and $N$. Shown are significant networks of genes relating to cell-to-cell signalling $(A)$, gastrointestinal pathways (B), amino acid metabolism (C) and lipid metabolism (D). $N=6$ for each line (3 control birds pooled from each time point) Genes or nodes coloured red are upregulated in colonised line $\mathrm{N}$ birds whereas those in green are upregulated in colonised line $\sigma_{1}$ birds.

Additional file 9: Figure. S5. IPA of DEGs identified between control birds of line $6_{1}$ and N. Shown are significant networks associated with predicted upstream regulators of DEGs: BCR (A), mir155 (B) and NFAT (C). Genes or nodes coloured red are upregulated in line $N$ birds whereas those in green are upregulated in line $\sigma_{1}$ birds. $N=6$ for each line ( 3 birds pooled from each time point)

Additional file 10: Table S5. STAR Alignment statistics for RNA-Seq transcript reads. 16: line $6_{1}$, In: line $N$, i: infected, c: control, b: bird, dpi: days post-infection.

Additional file 11: Table S6. Primer sequences for qRT-PCR

\section{Acknowledgements}

We thank animal attendants at the Moredun Research Institute and technicians at Edinburgh Genomics for their assistance.

\section{Authors' contributions}

KMR: designed and carried out the study, performed transcriptomic analysis and co-wrote the manuscript. JS: assisted with downstream analysis and editing the manuscript. AB: qRT-PCR validation and co-wrote the manuscript. CCU: assisted with sample collection. LV and AP were co-investigators of one of the grants that funded the work, provided critical analysis of findings and edited the manuscript. MS: conceived the study, secured funding and edited the manuscript. All authors approved the final version of the manuscript.

\section{Funding}

We gratefully acknowledge the support of the Scottish Government funding via the Rural \& Environmental Science and Analytical Services programme of research for 2016-2021 and the Biotechnology \& Biological Sciences 
Research Council (Institute Strategic Programme BBS/E/D/20002172 and Core Capability Grant in support of the National Avian Research Facility).

\section{Availability of data and materials}

Sequencing data have been submitted to the European Nucleotide Archive (https://www.ebi.ac.uk/ena) under accession number PRJEB24399.

\section{Declarations}

\section{Ethics approval}

All procedures were conducted under Home Office project licence PCD70CB48 in full compliance with the requirements of the Animal (Scientific Procedures) Act 1986, with the approval of the Moredun Institute Animal Welfare and Ethical Review Board. Housing and husbandry complied with the Code of Practice for Housing and Care of Animals Bred, Supplied or Used for Scientific Purposes and were also overseen by the Moredun Institute Animal Welfare and Ethical Review Board. Animals were culled by a schedule one method authorized by the Animals (Scientific Procedures) Act 1986. This study was performed in compliance with the Animal Research: Reporting of In Vivo Experiments (ARRIVE) guidelines as defined by the National Centre for the Replacement, Refinement and Reduction of Animals in Research (NC3Rs).

\section{Consent for publication}

Not applicable.

\section{Competing interests}

The authors declare no conflicts of interest and no competing financial interests.

\section{Author details}

${ }^{1}$ The Roslin Institute and Royal (Dick) School of Veterinary Studies, University of Edinburgh, Easter Bush, Midlothian EH25 9RG, UK. ${ }^{2}$ The Royal Veterinary College, Hawkshead Lane, Hatfield, Hertfordshire AL9 7TA, UK.

Received: 1 February 2021 Accepted: 20 May 2021

Published online: 04 June 2021

\section{References}

1. Havelaar AH, Kirk MD, Torgerson PR, Gibb HJ, Hald T, Lake RJ, et al. World Health Organization global estimates and regional comparisons of the burden of foodborne disease in 2010. PLoS Med. 2015;12(12):e1001923.

2. PHE. Anon. In: Zoonoses report UK. UK: Public Health England; 2017.

3. Tam CC, Rodrigues LC, Viviani L, Dodds JP, Evans MR, Hunter PR, et al. Longitudinal study of infectious intestinal disease in the UK (IID2 study): incidence in the community and presenting to general practice. Gut. 2012; 61(1):69-77.

4. Tam CC, O'Brien SJ. Economic cost of Campylobacter, norovirus and rotavirus disease in the United Kingdom. PLoS One. 2016;11(2):e0138526.

5. Janssen R, Krogfelt KA, Cawthraw SA, van Pelt W, Wagenaar JA, Owen RJ. Host-pathogen interactions in Campylobacter infections: the host perspective. Clin Microbiol Rev. 2008;21(3):505-18.

6. EFSA Panel on Biological Hazards (BIOHAZ). Scientific Opinion on Campylobacter in broiler meat production: control options and performance objectives and/or targets at different stages of the food chain. EFSA J. 2011; 9(4):2105.

7. Skarp CPA, Hänninen ML, Rautelin HIK. Campylobacteriosis: the role of poultry meat. Clin Microbiol Infect. 2016;22(2):103-9.

8. Wagenaar JA, French NP, Havelaar AH. Preventing Campylobacter at the source: why is it so difficult? Clin Infect Dis. 2013;57(11):1600-6.

9. Jorgenson F, Charlett, A., Arnold, E., Swift, C., Elviss, N.C.: A microbiological survey of Campylobacter contamination in fresh whole UK-produced chilled chickens at retail sale. In. Edited by England PH; 2018.

10. Newell DG, Fearnley C. Sources of Campylobacter colonization in broiler chickens. Appl Environ Microbiol. 2003;69(8):4343-51. https://doi.org/10.112 8/AEM.69.8.4343-4351.2003.

11. Rosenquist H, Nielsen NL, Sommer HM, Nørrung B, Christensen BB. Quantitative risk assessment of human campylobacteriosis associated with thermophilic Campylobacter species in chickens. Int J Food Microbiol. 2003; 83(1):87-103. https://doi.org/10.1016/50168-1605(02)00317-3.
12. EFSA Panel on Biological Hazards (BIOHAZ), Koutsoumanis K, Allende A, Alvarez-Ordóñez A, Bolton D, Bover-Cid S, et al. Update and review of control options for Campylobacter in broilers at primary production. EFSA J. 2020;18(4):e06090.

13. Li X, Swaggerty C, Kogut M, Chiang H-I, Wang Y, He H, et al. The paternal effect of Campylobacter jejuni colonization in ceca in broilers. Poult Sci. 2008;87:1742-7.

14. Psifidi A, Kranis A, Rothwell LM, Bremner A, Russell K, Robledo D, et al. Quantitative trait loci and transcriptome signatures associated with avian heritable resistance to Campylobacter. Sci Rep. 2020;11:1623.

15. Li X, Swaggerty CL, Kogut MH, Chiang HI, Wang Y, Genovese KJ, et al. Gene expression profiling of the local cecal response of genetic chicken lines that differ in their susceptibility to Campylobacter jejuni colonization. PLoS One. 2010;5(7):e11827.

16. Li XY, Swaggerty CL, Kogut MH, Chiang HI, Wang Y, Genovese KJ, et al. Caecal transcriptome analysis of colonized and non-colonized chickens within two genetic lines that differ in caecal colonization by Campylobacter jejuni. Anim Genet. 2011;42(5):491-500.

17. Li X, Swaggerty C, Kogut M, Chiang H-I, Wang Y, He H, et al. Systemic response to Campylobacter jejuni infection by profiling gene transcription in the spleens of two genetic lines of chickens. Immunogenetics. 2011;64:59-69.

18. Connell S, Meade KG, Allan B, Lloyd AT, Downing T, O'Farrelly C, et al. Genome-wide association analysis of avian resistance to Campylobacter jejuni colonization identifies risk locus spanning the $\mathrm{CDH} 13$ gene. $\mathrm{G} 3$ (Bethesda). 2013;3(5):881-90.

19. Connell S, Meade KG, Allan B, Lloyd AT, Kenny E, Cormican P, et al. Avian resistance to Campylobacter jejuni colonization is associated with an intestinal immunogene expression signature identified by mRNA sequencing. PLoS One. 2012;7(8):e40409.

20. Boyd Y, Herbert EG, Marston KL, Jones MA, Barrow PA. Host genes affect intestinal colonisation of newly hatched chickens by Campylobacter jejuni. Immunogenetics. 2005;57(3-4):248-53.

21. Psifidi A, Fife M, Howell J, Matika O, van Diemen PM, Kuo R, et al. The genomic architecture of resistance to Campylobacter jejuni intestinal colonisation in chickens. BMC Genomics. 2016;17:293.

22. Swaggerty $\mathrm{CL}$, Pevzner IY, He H, Genovese KJ, Kogut MH. Selection for pro-inflammatory mediators produces chickens more resistant to Campylobacter jejuni. Poult Sci. 2017;96(6):1623-7.

23. Shaughnessy RG, Meade KG, Cahalane S, Allan B, Reiman C, Callanan JJ, et al. Innate immune gene expression differentiates the early avian intestinal response between Salmonella and Campylobacter. Vet Immunol Immunopathol. 2009;132(2-4):191-8.

24. Shaughnessy RG, Meade KG, McGivney BA, Allan B, O'Farrelly C. Global gene expression analysis of chicken caecal response to Campylobacter jejuni. Vet Immunol Immunopathol. 2011;142(1-2):64-71.

25. Smith CK, Abuoun M, Cawthraw SA, Humphrey TJ, Rothwell L, Kaiser P, et al. Campylobacter colonization of the chicken induces a proinflammatory response in mucosal tissues. FEMS Immunol Med Microbiol. 2008;54(1):114-21.

26. Reid WD, Close AJ, Humphrey S, Chaloner G, Lacharme-Lora L, Rothwell L, et al. Cytokine responses in birds challenged with the human food-borne pathogen Campylobacter jejuni implies a Th17 response. R Soc Open Sci. 2016;3(3):150541.

27. Humphrey S, Chaloner G, Kemmett K, Davidson N, Williams N, Kipar A, et al. Campylobacter jejuni is not merely a commensal in commercial broiler chickens and affects bird welfare. mBio. 2014;5(4):e01364-14.

28. Fife MS, Howell JS, Salmon N, Hocking PM, van Diemen PM, Jones MA, et al. Genome-wide SNP analysis identifies major QTL for Salmonella colonization in the chicken. Anim Genet. 2011:42(2):134-40.

29. Chintoan-Uta C, Wisedchanwet T, Glendinning L, Bremner A, Psifidi A, Vervelde $L$, et al. Role of cecal microbiota in the differential resistance of inbred chicken lines to colonization by Campylobacter jejuni. Appl Environ Microbiol. 2020;86(7):e02607-19.

30. Subramanian A, Tamayo P, Mootha VK, Mukherjee S, Ebert BL, Gillette MA, et al. Gene set enrichment analysis: a knowledge-based approach for interpreting genome-wide expression profiles. Proc Natl Acad Sci. 2005; 102(43):15545-50

31. Krämer A, Green J, Pollard J Jr, Tugendreich S. Causal analysis approaches in ingenuity pathway analysis. Bioinformatics. 2014;30(4):523-30.

32. Livigni A, O'Hara L, Polak ME, Angus T, Wright DW, Smith LB, et al. A graphical and computational modeling platform for biological pathways. Nat Protoc. 2018;13(4):705-22. 
33. Vohra P, Chintoan-Uta C, Terra VS, Bremner A, Cuccui J, Wren BW, et al. Evaluation of glycosylated FlpA and SodB as subunit vaccines against Campylobacter jejuni colonisation in chickens. Vaccines (Basel). 2020;8(3):520.

34. Chaloner G, Wigley P, Humphrey S, Kemmett K, Lacharme-Lora L, Humphrey $T$, et al. Dynamics of dual infection with Campylobacter jejuni strains in chickens reveals distinct strain-to-strain variation in infection ecology. Appl Environ Microbiol. 2014;80(20):6366-72.

35. Ringoir DD, Korolik V. Colonisation phenotype and colonisation potential differences in Campylobacter jejuni strains in chickens before and after passage in vivo. Vet Microbiol. 2003;92(3):225-35.

36. Young CR, Ziprin RL, Hume ME, Stanker LH. Dose response and organ invasion of day-of-hatch Leghorn chicks by different isolates of Campylobacter jejuni. Avian Dis. 1999;43(4):763-7.

37. Cawthraw SA, Wassenaar TM, Ayling R, Newell DG. Increased colonization potential of Campylobacter jejuni strain 81116 after passage through chickens and its implication on the rate of transmission within flocks. Epidemiol Infect. 1996;117(1):213-5.

38. Kaufman J, Milne S, Göbel TWF, Walker BA, Jacob JP, Auffray C, et al. The chicken $B$ locus is a minimal essential major histocompatibility complex. Nature. 1999;401(6756):923-5.

39. Palyada K, Threadgill D, Stintzi A. Iron acquisition and regulation in Campylobacter jejuni. J Bacteriol. 2004;186(14):4714-29.

40. Smith J, Sadeyen JR, Paton IR, Hocking PM, Salmon N, Fife M, et al. Systems analysis of immune responses in Marek's disease virus-infected chickens identifies a gene involved in susceptibility and highlights a possible novel pathogenicity mechanism. J Virol. 2011;85(21):11146-58.

41. Patel MB, Oza NA, Anand IS, Deshpande SS, Patel CN. Liver x receptor: a novel therapeutic target. Indian J Pharm Sci. 2008;70(2):135-44.

42. Schultz JR, Tu H, Luk A, Repa JJ, Medina JC, Li L, et al. Role of LXRs in control of lipogenesis. Genes Dev. 2000;14(22):2831-8.

43. van Raalte DH, Li M, Pritchard PH, Wasan KM. Peroxisome proliferatoractivated receptor (PPAR)-alpha: a pharmacological target with a promising future. Pharm Res. 2004;21(9):1531-8.

44. A-Gonzalez N, Castrillo A. Liver $X$ receptors as regulators of macrophage inflammatory and metabolic pathways. Biochim Biophys Acta. 2011;1812(8): 982-94.

45. Wildenberg ME, van den Brink GR. FXR activation inhibits inflammation and preserves the intestinal barrier in IBD. Gut. 2011;60(4):432-3.

46. Armstrong LE, Guo GL. Role of FXR in liver inflammation during nonalcoholic steatohepatitis. Curr Pharmacol Rep. 2017;3(2):92-100.

47. Korbecki J, Bobiński R, Dutka M. Self-regulation of the inflammatory response by peroxisome proliferator-activated receptors. Inflamm Res. 2019; 68(6):443-58.

48. Zandbergen F, Plutzky J. PPARalpha in atherosclerosis and inflammation. Biochim Biophys Acta. 2007;1771(8):972-82.

49. Le Menn G, Neels JG. Regulation of immune cell function by PPARs and the connection with metabolic and neurodegenerative diseases. Int J Mol Sci. 2018;19(6):1575.

50. Azuma YT, Nishiyama K, Matsuo Y, Kuwamura M, Morioka A, Nakajima H, et al. PPARa contributes to colonic protection in mice with DSS-induced colitis. Int Immunopharmacol. 2010;10(10):1261-7.

51. Klepsch V, Moschen AR, Tilg H, Baier G, Hermann-Kleiter N. Nuclear receptors regulate intestinal inflammation in the context of IBD. Front Immunol. 2019;10:1070.

52. Barcia AM, Harris HW. Triglyceride-rich lipoproteins as agents of innate immunity. Clin Infect Dis. 2005;41(Suppl 7):S498-503.

53. Kong J, Zhang Z, Musch MW, Ning G, Sun J, Hart J, et al. Novel role of the vitamin $D$ receptor in maintaining the integrity of the intestinal mucosal barrier. Am J Physiol Gastrointest Liver Physiol. 2008;294(1):G208-16.

54. Yin K, Agrawal DK. Vitamin D and inflammatory diseases. J Inflamm Res. 2014;7:69-87.

55. Mousavi S, Lobo de Sá FD, Schulzke JD, Bücker R, Bereswill S, Heimesaat MM. Vitamin D in acute Campylobacteriosis-results from an intervention study applying a clinical Campylobacter jejuni induced enterocolitis model. Front Immunol. 2019;10:2094

56. Mahesh G, Biswas R. MicroRNA-155: a master regulator of inflammation. J Interf Cytokine Res. 2019;39(6):321-30

57. Liu X, Liu L, Zhang M, Wang H, Yang N, Li X. Chicken cecal microRNAs in the response to Campylobacter jejuni inoculation by Solexa sequencing. Poult Sci. 2016;95(12):2819-23.
58. Barrow PA, Bumstead N, Marston K, Lovell MA, Wigley P. Faecal shedding and intestinal colonization of Salmonella enterica in in-bred chickens: the effect of host-genetic background. Epidemiol Infect. 2004;132(1):117-26.

59. Calenge F, Vignal A, Demars J, Fève K, Menanteau P, Velge P, et al. New QTL for resistance to Salmonella carrier-state identified on fowl microchromosomes. Mol Gen Genomics. 2011;285(3):237-43.

60. Calenge F, Lecerf F, Demars J, Feve K, Vignoles F, Pitel F, et al. QTL for resistance to Salmonella carrier state confirmed in both experimental and commercial chicken lines. Anim Genet. 2009;40(5):590-7.

61. Tilquin P, Barrow PA, Marly J, Pitel F, Plisson-Petit F, Velge P, et al. A genome scan for quantitative trait loci affecting the Salmonella carrier-state in the chicken. Genet Sel Evol. 2005;37(5):539-61.

62. Swaggerty $C L$, Kogut $M H$, Ferro PJ, Rothwell L, Pevzner IY, Kaiser P. Differential cytokine mRNA expression in heterophils isolated from Salmonella-resistant and -susceptible chickens. Immunology. 2004;113(1): 139-48.

63. Byrne CM, Clyne M, Bourke B. Campylobacter jejuni adhere to and invade chicken intestinal epithelial cells in vitro. Microbiology. 2007;153(2):561-9.

64. Bailey RA, Kranis A, Psifidi A, Watson KA, Rothwell L, Hocking PM, et al. Colonization of a commercial broiler line by Campylobacter is under limited genetic control and does not significantly impair performance or intestinal health. Poult Sci. 2018.

65. Ni L, Fang P, Hu Z-L, Zhou H-Y, Chen J-G, Wang F, et al. Identification and function of acid-sensing ion channels in RAW 264.7 macrophage cells. Current Medical Science. 2018;38(3):436-42.

66. Kong $X$, Tang $X$, Du W, Tong J, Yan Y, Zheng F, et al. Extracellular acidosis modulates the endocytosis and maturation of macrophages. Cell Immunol. 2013;281(1):44-50

67. Miller MM, Taylor RL Jr. Brief review of the chicken major histocompatibility complex: the genes, their distribution on chromosome 16, and their contributions to disease resistance. Poult Sci. 2016;95(2):375-92.

68. Shiina T, Briles WE, Goto RM, Hosomichi K, Yanagiya K, Shimizu S, et al. Extended gene map reveals tripartite motif, C-type lectin, and lg superfamily type genes within a subregion of the chicken MHC-B affecting infectious disease. J Immunol. 2007;178(11):7162-72.

69. Friis C, Wassenaar TM, Javed MA, Snipen L, Lagesen K, Hallin PF, et al. Genomic characterization of Campylobacter jejuni strain M1. PLoS One. 2010;5(8):e12253.

70. Andrews S: FastQC: a quality control tool for high throughput sequence data. Available online at: https://www.bioinformatics.babraham.ac.uk/ projects/fastqc. 2010.

71. Martin M. Cutadapt removes adapter sequences from high-throughput sequencing reads. J Comput Biol. 2011;17(1):3.

72. Dobin A, Davis CA, Schlesinger F, Drenkow J, Zaleski C, Jha S, et al. STAR: ultrafast universal RNA-seq aligner. Bioinformatics. 2013;29(1):15-21.

73. Liao Y, Smyth GK, Shi W. FeatureCounts: an efficient general purpose program for assigning sequence reads to genomic features. Bioinformatics. 2014;30(7):923-30

74. Dennis G, Sherman BT, Hosack DA, Yang J, Gao W, Lane HC, et al. DAVID: database for annotation, visualization, and integrated discovery. Genome Biol. 2003:4(9):R60.

75. Pfaffl MW. A new mathematical model for relative quantification in real-time RT-PCR. Nucleic Acids Res. 2001;29(9):e45.

\section{Publisher's Note}

Springer Nature remains neutral with regard to jurisdictional claims in published maps and institutional affiliations.

\section{Ready to submit your research? Choose BMC and benefit from}

- fast, convenient online submission

- thorough peer review by experienced researchers in your field

- rapid publication on acceptance

- support for research data, including large and complex data types

- gold Open Access which fosters wider collaboration and increased citations

- maximum visibility for your research: over $100 \mathrm{M}$ website views per year

At $\mathrm{BMC}$, research is always in progress.

Learn more biomedcentral.com/submissions 